\title{
COVID CONCEPT
}

\section{New Bullous Eruptions in a COVID-19 Positive Patient in an Intensive Care Unit}

\author{
Angela Kim, DO, MPH${ }^{1}$, Muneeb Khan, MD², Ann Lin, DO, MS ${ }^{1}$, Hongbei Wang, MD, $\mathrm{PhD}^{3}$, \\ Louis Siegel, DO ${ }^{1}$, Suzanne Sirota-Rozenberg, DO ${ }^{1}$ \\ ${ }^{1}$ Division of Dermatology, St. John's Episcopal Hospital, Far Rockaway, NY \\ 2Department of Family Medicine, St. John's Episcopal Hospital, Far Rockaway, NY \\ ${ }^{3}$ Department of Dermatopathology, Hofstra Northwell School of Medicine, Hempstead, NY
}

\section{ABSTRACT}

Bullous pemphigoid (BP) is an autoimmune blistering disorder that typically occurs in older adults. The etiopathogenesis of BP is unclear, although viral triggers have been reported. ${ }^{1}$ We present a case of new onset pauci-cellular BP in a patient admitted to Intensive Care Unit from an acute COVID-19 infection. New onset bullous eruptions in the setting of COVID-19 infection should elicit suspicions and consider the differential diagnosis of BP.

\section{INTRODUCTION}

Bullous pemphigoid (BP) is an autoimmune blistering disorder that most commonly affects older adults and is characterized by tense bullae. Although most cases of BP have no precipitating factors, virus-induced BPs have been reported. ${ }^{1}$ We describe a case of new onset BP in a patient with acute COVID-19 infection during the pandemic peak in New York.

\section{CASE PRESENTATION}

A 76-year-old male from a nursing home with a past medical history of hypertension, diabetes mellitus, hyperlipidemia, and major depressive disorder presented to the emergency department with altered mental status, hypotension, tachycardia, hypoxia, and a fever of 100.9F. A positive COVID-19 polymerase chain reaction, labs with elevated D-dimer, lactate dehydrogenase, ferritin, C-reactive protein, and erythrocyte sedimentation rate, and a chest X-ray showing bilateral infiltrates, were all consistent with progressing COVID-19 respiratory syndrome. Following respiratory failure, the patient was intubated and admitted to the Intensive Care Unit. Patient's home medications were discontinued at admission.

Hydroxychloroquine and azithromycin were initiated per hospital protocol, along with intravenous methylprednisolone and anakinra. There were no skin findings initially. On day 3 , multiple $2 \mathrm{~mm}$ to $6 \mathrm{~cm}$ tense bullae developed on his left arm(Figure 1). A shave biopsy of a $2 \mathrm{~mm}$ intact bullae for Hematoxylin \& Eosin(H\&E) and a $3 \mathrm{~mm}$ perilesional punch biopsy for direct immunofluorescence(DIF) were performed. The H\&E stain(Figure 2) showed a cell-poor subepidermal bulla, while DIF(Figure 3) revealed linear

May 2021 Volume 5 Issue 3 


\section{SKIN}

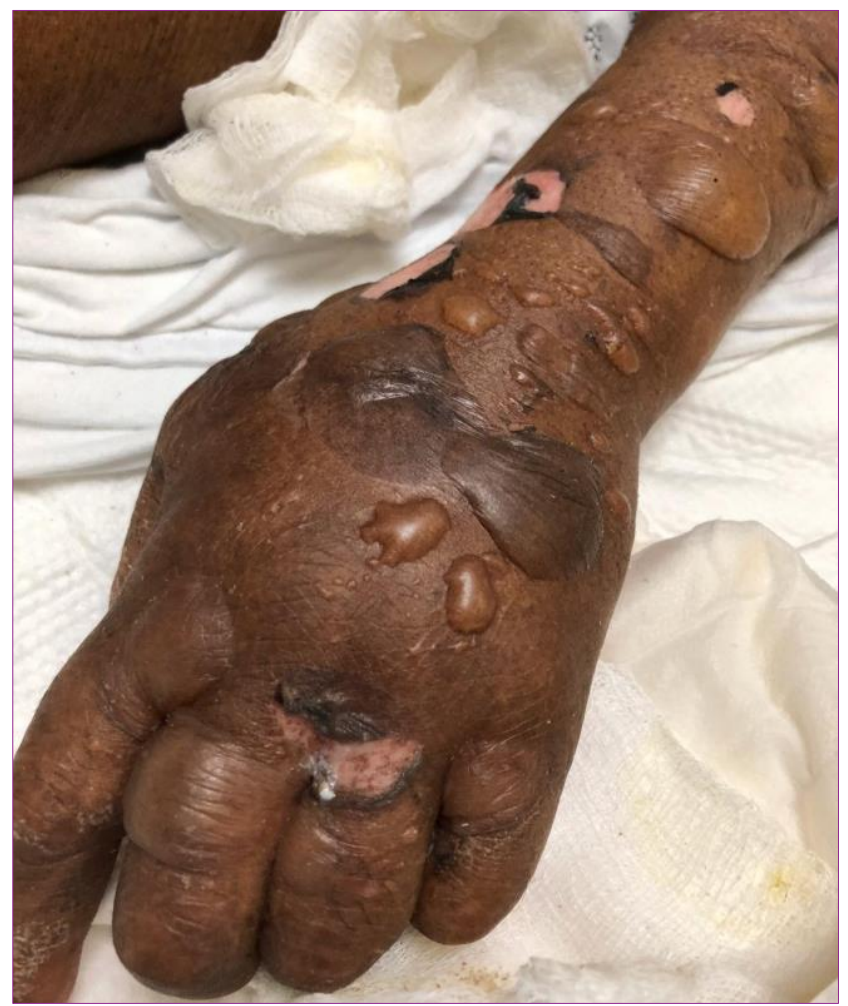

Figure 1. Bullous Pemphigoid. Clinical presentation of tense and flaccid bullae with erosions on left upper extremity

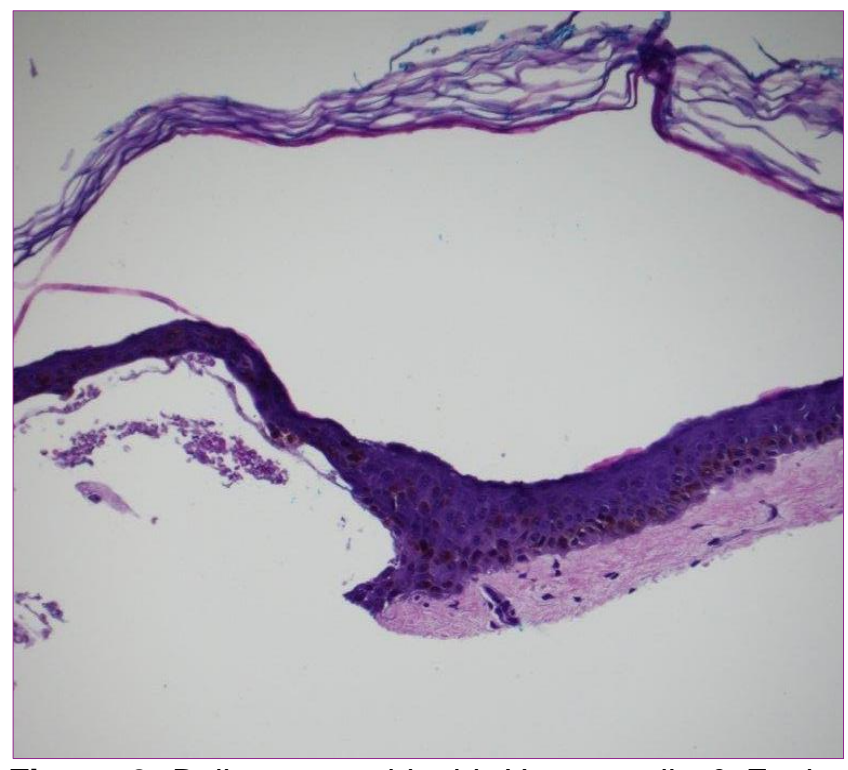

Figure 2. Bullous pemphigoid. Hematoxylin \& Eosin histologic examination of biopsy specimen showing a pauci-cellular subepidermal bullae with minimal infiltrate

Immunoglobulin $\mathrm{G}(\lg G)$ at the dermoepidermal junction. Bullae were ruptured with a sterile needle, and wound care was rendered with twice-daily applications of topical betamethasone dipropionate, augmented $0.05 \%$ ointment. No new blisters recurred. Patient recovered overall and was extubated and discharged.

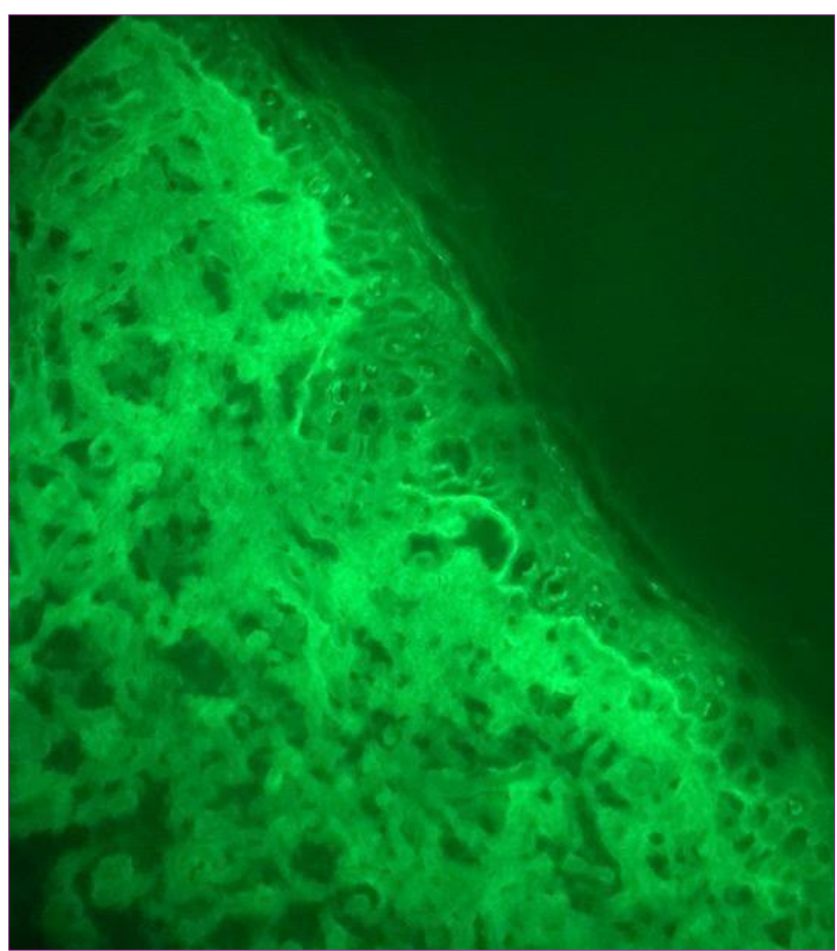

Figure 3. Bullous Pemphigoid. Direct immunofluorescence image of perilesional punch biopsy specimen showing linear $\lg G$

\section{DISCUSSION}

The etiopathogenesis of BP is complex and not yet fully understood, although viral infections with HIV, EBV, CMV, HHV, HHV6, HBV, and HCV have been reported to trigger BP. ${ }^{1}$ A report of three pediatric cases describes post-immunization and post-viral induced BP. ${ }^{2}$ To date, there is little information on the association between severe acute respiratory syndrome coronavirus 2 (SARS-CoV-2) virus and BP and more studies are needed. 
Without a history of BP, our intubated patient was non-verbal at the time of examination and therefore was unable to report any prodromal skin pruritus or tenderness. New onset bullous eruptions occurred while in critical care for COVID-19 and despite being on a systemic steroid. Cutaneous symptoms of SARS-CoV-2 are reported to present as petechial-, erythematous-, urticarial-, and pernio-like rashes, ${ }^{3}$ which our patient did not have. Although the patient fit the usual age group for new BP onset, the mechanism of induction, especially in the setting of SARSCoV-2, remains unclear.

On day 1-2 of admission, the patient received $2 \mathrm{~L}$ of fluids/day and was oliguric, but urine output normalized on day 3 when skin eruptions occurred. Of note, the right upper extremity and lower extremities were not edematous and unaffected throughout admission. Given these facts and the paucicellar subepidermal bullae, a differential diagnosis of edema blisters must be considered. Edema blisters are generally observed on lower extremities and are associated with chronic venous insufficiency, congestive heart failure, hepatic or renal failures, which were negative in our patient. ${ }^{4}$ Although paucicellular, our H\&E did not show any epidermal spongiosis or dermal edema, which is evident in edema blisters.

Typically, eosinophils are the defining inflammatory infiltrates in BP. Although pauci-cellular BP is uncommon, studies have demonstrated IgG depleting BP180 in the hemidesmosomes and weakening the lamina lucida without complement activation in BP. 5,6 This may explain the minimal inflammation within the bulla and positive IgG on DIF in our case. Other reasons may include systemic steroid received since admission or biopsy specimen size not ample enough to visualize eosinophils.

Most BP DIF shows IgG and/or C3 in the basement membrane zone. The sensitivity of DIF in BP ranges 88.3-84\% additionally, in a large group of BP patients, DIF detected IgG was $91.4 \%$ and $73.6 \%$ for C3. Isolated IgG deposition was $19.8 \%{ }^{8}$ Our patient showed a unique case of BP with positive IgG but negative for C3, $\lg A$, and IgM on DIF. False positive DIF can occur if biopsies are taken from lower extremities or from the bullae itself, but our DIF was taken from the upper extremity, the only location involved, and from an uninvolved perilesional skin within $1 \mathrm{~cm}$ from a blister. Correlating DIF with ELISA testing for anti-BP180 and anti-BP230 antibodies and/or indirect immunofluorescence(IIF) may have been helpful. However, people without BP can also have positive autoantibodies on ELISA. ELISA and IIF were not performed on our patient when dermatopathology resulted, which was about a week after the biopsy, as lesions resolved and it did not change our management.

A cell-poor subepidermal blister and linear IgG on DIF may be seen in epidermolysis bullosa acquisita(EBA). However, EBA typically occurs in a younger age group, on sites of trauma, and heals with scarring, dyspigmentation, and milia formation. Bullous eruptions on our patient occurred on day 3 without any previous or current IV access on the affected extremity. Furthermore, there was no trauma reported or observed on the physical exam. Upon topical treatment, all lesions on our patient resolved and healed without scarring. Although our patient had a history of diabetes, positive DIF ruled out bullous diabeticorum, edema blister, and coma blister, which may have negative DIF or positive IgM and C3.4

May 2021 Volume 5 Issue 3 
Drug-induced BP, which is triggered by a combination of genetic predisposition and inciting medications, can be considered. The exact mechanism is not well elucidated, but it leads to alteration of the immune response to the epidermal basement membrane..$^{9}$ Our patient's long-term home medications were discontinued at admission until discharge and were non-contributory. A drug chart while inpatient was created, and no inciting medications commonly associated with BP were given. Initially, a dose of vancomycin and piperacillin/tazobactam each was given. Vancomycin can induce linear IgA bullous dermatosis but DIF would show linear IgA deposition, which was negative in our patient. No report has shown association between $\mathrm{BP}$ and hydroxychloroquine or azithromycin.

Bullous eruption only occurred on our patient's left upper extremity. Other unilateral COVID related rashes have been reported, ${ }^{10,11}$ and reasons behind its localized presentations are still unclear. A report of a unilateral BP on one leg with chronic venous stasis, speculates that inflammation from stasis may have triggered the BP. ${ }^{12}$ Inflammation, as evidenced by serum markers, may have contributed to our bullous eruption.

Immunosuppressives and anti-inflammatory medications are mainstay therapies for BP. In our case, potent topical corticosteroid resolved our localized BP when the systemic corticosteroid for the respiratory disease did not help. More studies are needed to understand the complexities of SARS-CoV-2 and BP separately and together. When encountering new bullous eruptions in patients with active SARS-COV-2, clinicians should take caution and consider BP in their differential diagnosis as timely diagnosis and management can improve patient outcome.
Acknowledgement: We would like to thank Dr. Sadaf Sheikh (Department of Pathology, St. John's Episcopal Hospital, Far Rockaway, NY) for providing the H\&E pathology photos for our case

Conflict of Interest Disclosures: None

Funding: None

Corresponding Author:

Angela Kim, DO, MPH

Division of Dermatology

St. John's Episcopal Hospital

327 Beach 19th Street

Far Rockaway, New York, 11691

Phone: 718-869-7108

Fax: 408-827-9056

Email: angelakim86@gmail.com

References:

1. Jang $\mathrm{H}$, Jin $\mathrm{YJ}$, Yoon $\mathrm{CH}$, et al. Bullous pemphigoid associated with chronic hepatitis $\mathrm{C}$ virus infection in a hepatitis $B$ virus endemic area: A case report. Medicine (Baltimore). 2018;97(15):e0377. doi:10.1097/MD.0000000000010377

2. Baroero L, Coppo P, Bertolino L, et al. Three case reports of post immunization and post viral Bullous Pemphigoid: looking for the right trigger. BMC Pediatr. 2017;17(1):60. Published 2017 Feb 23. doi:10.1186/s12887-017-0813-0

3. Ortega-Quijano D, Jimenez-Cauhe J, SeldaEnriquez $\mathrm{G}$, et al. Algorithm for the classification of Covid-19 rashes, Journal of the American Academy of Dermatology (2020), doi: https://doi.org/10.1016/j.jaad.2020.05.034.

4. Sami N, Yeh SW, Ahmed AR. Blistering diseases in the elderly: diagnosis and treatment. Dermatol Clin. 2004 Jan;22(1):73-86. doi: 10.1016/s07338635(03)00116-5. PMID: 15018011. Freeman EE, McMahon DE, Lipoff JB, et al.

5. Hiroyasu S, Ozawa T, Kobayashi $\mathrm{H}$, et al. Bullous pemphigoid IgG induces BP180 internalization via a macropinocytic pathway. Am J Pathol. 2013;182(3):828-840. doi:10.1016/j.ajpath.2012.11.029

6. Iwata $\mathrm{H}$, Kamio N, Aoyama $\mathrm{Y}$, et al. IgG from patients with bullous pemphigoid depletes cultured keratinocytes of the $180-\mathrm{kDa}$ bullous pemphigoid antigen (type XVII collagen) and weakens cell attachment. J Invest Dermatol. 2009;129(4):919-926. doi:10.1038/jid.2008.305

7. Buch AC, Kumar H, Panicker N, Misal S, Sharma Y, Gore CR. A Cross-sectional Study of Direct

May 2021 Volume 5 Issue 3 
Immunofluorescence in the Diagnosis of

Immunobullous Dermatoses. Indian J Dermatol.

2014 Jul;59(4):364-8. doi: 10.4103/0019-

5154.135488. PMID: 25071256; PMCID:

PMC4103273.

8. Meijer JM, Diercks GFH, de Lang EWG, Pas HH, Jonkman MF. Assessment of Diagnostic Strategy for Early Recognition of Bullous and Nonbullous

Variants of Pemphigoid. JAMA Dermatol. 2019

Feb 1;155(2):158-165. doi:

10.1001/jamadermatol.2018.4390. PMID:

30624575 ; PMCID: PMC6439538.

9. Verheyden MJ, Bilgic A, Murrell DF. A Systematic Review of Drug-Induced Pemphigoid. Acta Derm

Venereol. 2020 Aug 17;100(15):adv00224. doi: 10.2340/00015555-3457. PMID: 32176310.

10. Glick LR, Fogel AL, Ramachandran S, Barakat

LA. Unilateral laterothoracic exanthem in association with coronavirus disease 2019. JAAD Case Rep. 2020 Sep;6(9):900-901. doi:

10.1016/j.jdcr.2020.07.020. Epub 2020 Jul 21.

PMID: 32835047; PMCID: PMC7372280.

11. Karaca Z, Yayli S, Çalışkan O. A unilateral purpuric rash in a patient with COVID-19 infection. Dermatol Ther. 2020 Jul;33(4):e13798. doi: 10.1111/dth.13798. Epub 2020 Jul 8. PMID: 32530130; PMCID: PMC7300488.

12. Shi, C. R., Charrow, A., Granter, S. R., Christakis, A., \& Wei, E. X. (2018). Unilateral, localized bullous pemphigoid in a patient with chronic venous stasis. JAAD case reports, 4(2), 162-164.

https://doi.org/10.1016/j.jdcr.2017.09.032 